\title{
Flow thresholds of nonodorous air through the human naris as a function of temperature and humidity
}

\author{
VERNON A. BENIGNUS and JAMES D. PRAH \\ U.S. Environmental Protection Agency, University of North Carolina at Chapel Hill, \\ Chapel Hill, North Carolina 27514
}

\begin{abstract}
The process of olfactory sensation is probably a Gestalt consisting of motor, physical, and chemical components formed during the transport of the odor-bearing airstream past the olfactory epithelium. In studying a sensory process, it is often necessary to examine the components independently. Apart from its contribution to the olfactory Gestalt, in dynamic olfactometry airflow may distract from, compete with, or mask chemical sensation. Four nonsmoking male subjects were employed to obtain flow thresholds of nonodorous air at eight temperature/ humidity combinations. The stimulus-generating apparatus was housed in a temperatureregulated oven. All materials in contact with the airstream were thoroughly cleaned and were constructed of nonreactive materials to avoid olfactory cues. An evaluation of the quality of the output demonstrated purity. A Békésy technique was used to deliver stimuli via a nasal catheter to the subject. Thresholds were found to be nearly identical for the lowest humidity levels regardless of the temperature. In general, the closer the temperature/humidity was to physiological normal, the higher the threshold. Since the thresholds were so strongly functions of temperature and humidity, it is likely that transduction was by trigeminal thermoreceptive afferents. The utilization of subthreshold flows in olfactory research may reduce the trigeminal component in olfaction, thus permitting better assessment of the contribution of the olfactory nerve to olfactory sensation.
\end{abstract}

Before commencing olfactory experimentation utilizing a flow dilution olfactometer, it was thought necessary to determine the sensitivity of the human nose to purified airflow in order to avoid the possibility of confusion of airflow parameters with odorant stimulation. A search of the literature revealed no previous attempts to do so, although temperature, flow, and relative humidity (RH) have been evaluated as variables in olfactory sensitivity (Bocca \& Battiston, 1964; Grundvig, Dustman, \& Beck, 1967; Kerka \& Humphreys, 1956; LeMagnen, 1944-1945; Mullins, 1955; Rehn, 1978; Schneider, Costiloe, Vega, \& Wolf, 1963; Stone, 1963; Tucker, 1963). These investigators did not seek to characterize sensitivity to airflow per se.

There is probably more than one sensory mechanism capable of transducing the flow of purified air through the nose. The anterior ethmoid branch of the trigeminal nerve which innervates the nasal mucosa, but not the olfactory mucosa (Douek, 1974), is mechano- and thermoreceptive and capable of responding to the flow stimulus as mechanical distortion, cooling, or warming. At physiological levels of stimulation,

Reprint requests should be sent to James D. Prah, U.S. Environmental Protection Agency, Clinical Studies Division, Medical Research Building C, No. 224H, Mason Farm Road, University of North Carolina, Chapel Hill, North Carolina 27514. olfactory chemosensors are not mechanosensitive, but extreme levels of flow might produce sensation (Moulton \& Tucker, 1964).

The normal perception of odor may be best regarded as a Gestalt composed of a variety of physical, chemical, and motor components formed by the passage of the odor-bearing airstream through the nasal passages. In order to better understand the psychophysics of a perceptual system, it may be fruitful to simplify it by examining the components of the Gestalt independent of the Gestalt. To be sure, stimulation of the olfactory receptors in the absence of detectable air flow and muscle sensory feedback from inspiration disrupts the Gestalt, but it is not necessarily the Gestalt that is being studied. In order to assess the contribution of airflow to olfactory perception, it is desirable to determine the level of flow that can be sensed and the influence of temperature and RH on that airflow threshold.

The importance of the knowledge of these detection thresholds extends beyond the theoretical point of being a component in olfactory perception. From a purely practical point of view, experimenters may wish to select flow parameters which will not provide nonchemosensory cues that may distract from, compete with, or mask chemical sensation. Knowledge of the critical parameters of airflow detection can provide basic data for the design of apparatus and 
selection of flow values in an experimental design.

This study was executed to explore the sensitivity of the human nasal passages to the flow of purified air. Thermal stimulation is a function of the temperature of the air as well as the RH of the air. For these reasons, the threshold for the detection of nonodorized air was studied as functions of temperature and humidity.

\section{METHOD}

\section{Subjects}

Four nonsmoking male graduate students with a mean age of 27 years (range: $25-30$ years) were paid an hourly wage to participate. A physician examined all subjects to ensure good health. Two subjects participated at three temperature levels, one at two levels, and one at one level. Academic obligations precluded two of the subjects from completing all three temperature levels

\section{Apparatus}

The stimulus-generating apparatus (Figure 1) was housed in a large temperature-regulated oven (Thelco Model 28). To avoid olfactory cues, all parts of the apparatus that contacted the stimulus were constructed of nonreactive materials. These glass, 316 stainless steel, and Teflon parts were cleaned with chromic acid, then thoroughly rinsed, first in tap water and then in deionized water. The metering and shutoff valves were disassembled and cleaned in the same fashion to remove packing grease. After reassembly, the apparatus was checked for the absence of leaks. The stimulus source was compressed "zero" air with a hydrocarbon level of $<1 \mathrm{ppm}$. The air passed through a Pyrex glass wool $/$ silica gel/molsieve/activated charcoal/Pyrex glass wool filter, an electronic mass flow controller (Matheson Model 8240), and a second filter (Pyrex glass wool/drierite/activated charcoal/Pyrex glass wool). The Pyrex glass wool filters are not illustrated. The charcoal ensures air purity, and the other components remove moisture.

An analysis was conducted of the particulate level in the purified airstream after the second filter. A condensation nuclei monitor which responds to particles with diameters of $.002510^{-6} \mathrm{~m}$ and larger (Environment/One Corp. Model Rich 100) received direct input from the filter. This instrument, with a minimum resolution of $50 \mathrm{nuclei} / \mathrm{ml}$ indicated a particulate level of 60 to 150 nuclei $/ \mathrm{ml}$. By comparison, a HEPA filter was substituted and room air was drawn through by the monitor. The obtained particle count was 60 to 160 nuclei/ml. Even the cleanest air contains several hundred particles per milliliter (Air Quality Criteria for Particulate Matter, 1970).

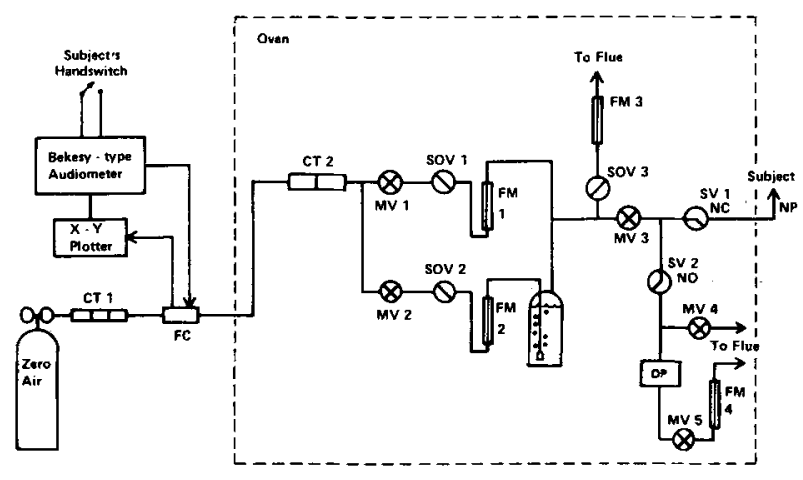

$\begin{array}{lllll}\text { CT - Clean-up trap } & \text { MV - Matering valve } & \text { SV - Solemoid valve } & \text { NC - Normally doted } & \text { NP - Nase pisce } \\ \text { FC - Ftaw controller } & \text { SOV - Shut off value } & \text { FM - Flow meter } & \text { NO - Normally open } & \text { DP - Dew point senso }\end{array}$

Figure 1. Schematic of the stimulus-generating apparatus.
Furthermore, a $.210^{-6} \mathrm{~m}$ pore size filter (Millipore No. FGLP. 04700) was attached to the output of the apparatus filter, and after flowing 2 liters/min of purified air through it for $5 \mathrm{~h}$, no measurable particulate matter was accumulated. A gas chromatographic analysis (Hewlett-Packard 7620A, Flame Ionization, $1.83 \mathrm{~m}, 10 \%$ Silar $10 \mathrm{C} 100 / 120$ mesh column) of the effluent of the filter revealed no detectable contaminant. While these data do not eliminate the possibility of contamination either by particulate matter or eluted chemicals, they do indicate that if any was present it was extremely low and was unlikely to have had a negative impact upon the quality of the purified airstream.

The conditioned air from the second filter was divided to provide a dry air line and a line to be humidified. Humidification was achieved by sparging the air through a 1-liter bottle nearly filled with deionized water. To attain maximum humidification, it was necessary to employ three sparge bottles in series. Humidified air was obtained from the headspace above the liquid. After humidification, the dry- and humid-air lines were rejoined and adjusted to yield the desired RH. A normally open solenoid valve (SV2) permitted continuous flow through the apparatus to maintain the preset RH level. A dew-point sensor (General Eastern Model 1100AP) monitored the RH. A flow meter (FM4) and metering valve (MV5) were used to keep a constant flow of $10 \mathrm{ml}$ through the sensor when measurements were taken, because at these temperatures the dew-point sensor was somewhat flow sensitive. The temperature was monitored via a thermocouple (Yellow Springs Instruments Model 46) attached to the effluent of FM4. When a stimulus was delivered, SV2 closed and SV1 opened, simultaneously delivering air to the subject.

To prevent stimulus heat loss, a hole was cut through the oven and a Styrofoam-paneled aluminum box was bolted to the outside of the oven. This permitted the box to become an extension of the oven and thereby maintain the stimulus delivery tube at the same temperature as the oven. The only part of this tube exposed to the environment was the Swagelok fitting that the nosepiece covered. Each subject was fitted with a stainless steel nosepiece shaped from a Swagelok fitting. The height of the nosepiece was constant $(1.2 \mathrm{~cm})$, but the width at the base ranged from 1.4 to 1.8 $\mathrm{cm}$. The width at the top varied from .8 to $1.2 \mathrm{~cm}$. These were designed to be inserted into the vestibule of each subject's nose no more than $1.2 \mathrm{~cm}$ and in a fashion that ensured an air-tight seal.

The subject was seated in an adjustable chair. The arm- and chinrests affixed to the oven were adjustable for comfort. Earphones were used to administer a low-level white noise to prevent auditory distraction. Visual distraction was avoided by the use of a screen. A small red-light-emitting diode within the visual field of the subject indicated the presence of a stimulus. One-second stimulus pulses occurred on a pseudorandom basis with the offtime averaging $2 \mathrm{sec}$ (range: 1.6 to $2.4 \mathrm{sec}$ ). It was discovered that regularly presented stimuli were monotonous and encouraged attentional lapses.

The rise time of the 1-sec pulse of air was $125 \mathrm{msec}$, and the fall time was $200 \mathrm{msec}$. This was determined oscillographically using a .001 -in.-diam iron-constantan thermocouple (Omega Model IRCO $001)$ inserted just inside the stimulus-delivery tube. The stimulus pulse was essentially a square wave with very little overshoot or tailing.

The flow controller was connected to an electrical output of a modified Békésy-type audiometer (Grason-Stadler Model 1701). This permitted the subject to vary the flow rate in the same manner as sound amplitude is varied in Békésy audiometry. As long as the subject maintained a response, the flow rate declined. When the hand-held response button (Grason-Stadler Model 1702-3500) was released, the flow rate increased until the subject again responded. The resulting flow oscillations were plotted on-line by an X-Y recorder and used to calculate approximate ascending and descending flow thresholds.

When lower thresholds were observed, a flow shunt was installed. This shunt consisted of a flow meter (FM3), a shut-off valve (SOV3), and a check valve (not illustrated) between SOV3 and the main line. The purpose of the shunt was to reduce the 


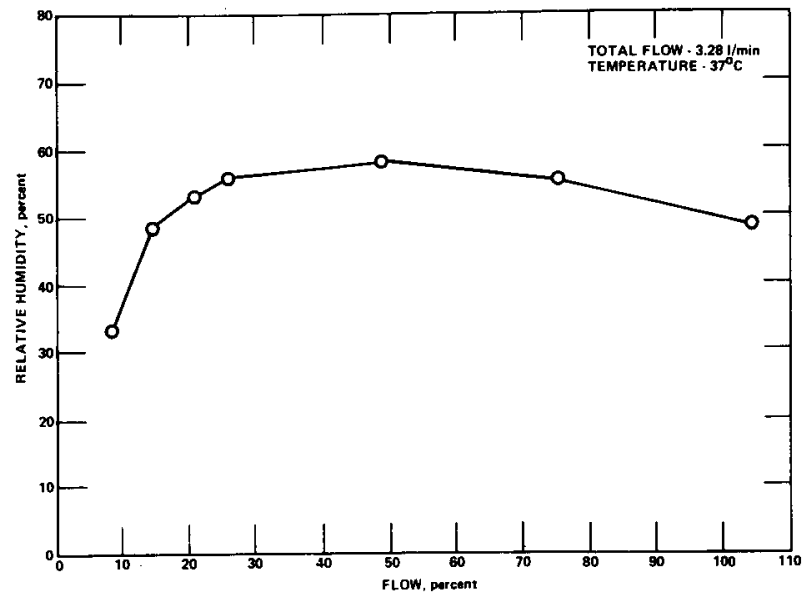

Figure 2. Stability of relative humidity at various flow rates.

flow to the subject while keeping a higher flow through the rest of the apparatus. Thus, the maximum flow the subject could experience would be, for example, one-fourth the normal flow, the remaining three-fourths being expended into the flue.

Three humidity ranges were used: $\mathrm{RH}<5 \%, 40 \%-55 \%$ RH, RH $>75 \%$. For convenience, these will be called RH $5 \%$, RH $50 \%$, and RH $75 \%$, respectively. These were chosen to encompass a wide humidity range. Three temperature levels were used: $32^{\circ}$, $37^{\circ}$, and $42^{\circ} \mathrm{C}$. These are body temperature $\pm 5^{\circ} \mathrm{C}$. All temperaturehumidity combinations were employed except that the combination of $42^{\circ} \mathrm{C}$ and $\mathrm{RH} 75 \%$ was not used because of discomfort reported by subjects in a preliminary study. The stability of the oven was $\pm .15^{\circ} \mathrm{C}$. Figure 2 illustrates the variability of the $\mathrm{RH}$ at an initial set point of $\mathrm{RH} 50 \%$ at a maximum flow rate of $3.28 \mathrm{liters} / \mathrm{min}$. This variation was due to several factors, including flow velocity at high flow rates and low air volume due to water pressure in the sparge bottle at low flow rates. Figure 2 also illustrates the conditions that brought about the usage of the flow shunt. A subject responding to low flow rates at RH $50 \%$ would often reduce the flow rate to such an extent that the RH level was significantly altered and the subject began responding to a lower RH. The flow shunt prevented this by reducing the flow available to the subject while maintaining the $\mathrm{RH}$ level.

\section{Procedure}

After the experimental procedure was explained to the subject, the naris with the lesser flow resistance was ascertained by having the subject breathe upon a mirror. This was determined daily so that the experiment was consistently begun in the naris with the lesser flow resistance. The nosepiece was positioned with respect to the chinrest, and the subjects were trained to maintain proper contact and position. The subjects were trained to breathe slowly and gently through the mouth while maintaining velopharyngeal closure. With this technique, the air flow entered one naris and exited via the other naris. Other methods could have been used, but this technique seemed, in preliminary studies, to produce the most stable results, to be the most easily learned, and the most comfortable. The subjects were instructed to depress the handheld response button as long as airflow pulses coincident with illumination of the indicator light could be detected. When airflow pulses could no longer be detected, the subject released the response button. Each of the three runs was begun at a suprathreshold level. These 13-min runs of about 390 stimuli each were separated by 5 -min breaks. The subjects participated 5 days per week at the same time each day. Each was run until his performance reached criterion. Criterion was defined as each run having approximately the same mean and variation in excursion of the ascending and descending tracking. It typically took several weeks to attain this degree of stability at a given temperature and humidity combination. The subjects were kept informed of the stimulus parameters and shown their performance after the experimental session was terminated. This maintained good cooperation and rapport.

\section{RESULTS}

Flow threshold (RL) was determined as the averaged midpoint of the ascending and descending flow oscillations of the criterion runs. Figure 3 presents a graph of the RL means and ranges across subjects for each RH level at the various temperature values. Ranges, rather than standard deviations, were employed as measures of dispersion to elucidate the degree of overlap between treatments. The lack of overlap obviates the need for significance testing.

The temperature and RH of the stimulus and the mean RL are shown for each subject in Table 1. As can be seen in Figure 3, the lowest RLs were uniformly observed at all temperature combinations with a RH of $5 \%$. RLs at these stimulus combinations exhibited a narrow range of values. Sensitivity to flow decreased as the temperature and humidity approached the physiologic norm. Sensitivity to flow at the $32^{\circ} \mathrm{C} / 50 \%$ combination was not markedly different from that of the RH 5\% combinations. Apparently, the low temperature coupled with evaporative cooling resulted in greater sensitivity to this temperature combination with RH $50 \%$. It is of interest to note that the maximum $R L$ was well below the normal physiologic level for inspired air of a resting human.

Only nominal differences were noted between the ascending and descending RLs. A comparison of the

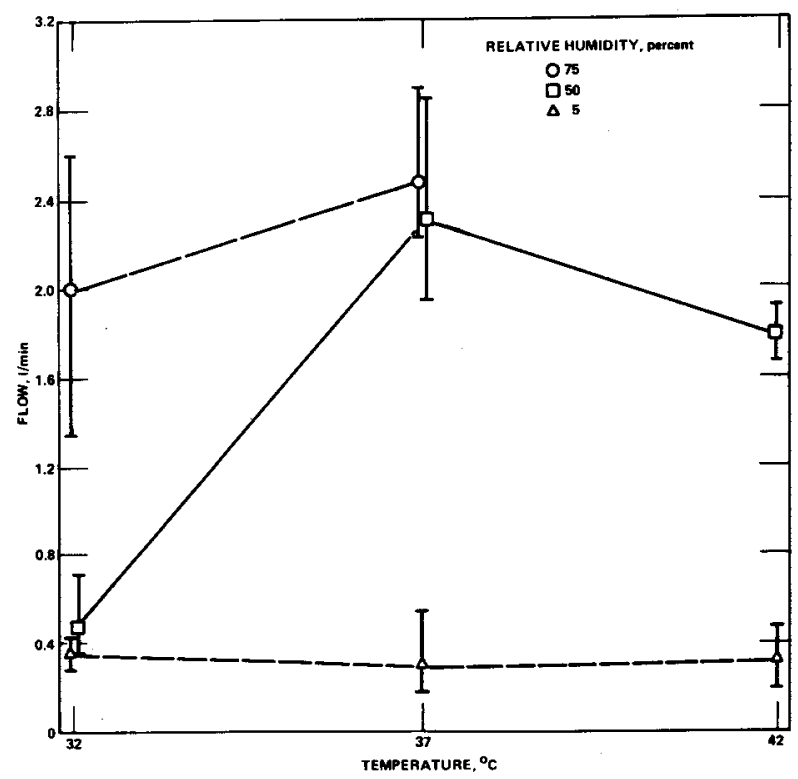

Figure 3. Plot of mean RL values and individual subject ranges. 
Table 1

Mean Flow RL in $L / M i n$ at the Various Temperature-Humidity Combinations

\begin{tabular}{|c|c|c|c|c|c|c|c|}
\hline \multirow[b]{2}{*}{${ }^{\circ} \mathrm{C}$} & \multirow[b]{2}{*}{$\mathrm{S}$} & \multicolumn{2}{|c|}{$\mathrm{RH}<5$} & \multicolumn{2}{|c|}{$\mathrm{RH}=40-55$} & \multicolumn{2}{|c|}{$\mathrm{RH}>75$} \\
\hline & & RL & $\mathbf{N}$ & RL & $\mathbf{N}$ & $\mathrm{RL}$ & $\mathbf{N}$ \\
\hline 32 & $\begin{array}{l}2 \\
5 \\
6\end{array}$ & $\begin{array}{l}.300 \\
.405 \\
.315\end{array}$ & $\begin{array}{l}85 \\
75 \\
59\end{array}$ & $\begin{array}{l}.387 \\
.723 \\
.362\end{array}$ & $\begin{array}{r}79 \\
111 \\
45\end{array}$ & $\begin{array}{l}1.343 \\
2.599 \\
2.050\end{array}$ & $\begin{array}{l}71 \\
53 \\
63\end{array}$ \\
\hline 37 & $\begin{array}{l}1 \\
2 \\
5 \\
6\end{array}$ & $\begin{array}{l}.546 \\
.201 \\
.196 \\
.284\end{array}$ & $\begin{array}{l}69 \\
81 \\
67 \\
87\end{array}$ & $\begin{array}{l}2.234 \\
2.154 \\
2.849 \\
1.943\end{array}$ & $\begin{array}{l}69 \\
47 \\
67 \\
67\end{array}$ & $\begin{array}{l}2.895 \\
2.224 \\
2.252\end{array}$ & $\begin{array}{r}111 \\
61\end{array}$ \\
\hline 42 & $\begin{array}{l}2 \\
5\end{array}$ & $\begin{array}{l}.479 \\
.191\end{array}$ & $\begin{array}{r}109 \\
83\end{array}$ & $\begin{array}{l}1.665 \\
1.908\end{array}$ & $\begin{array}{l}81 \\
89\end{array}$ & & \\
\hline
\end{tabular}

Note-S subject number; $N=$ the number of ascending and descending trials used to determine the $R L$.

first and third 13-min criterion runs for each subject revealed no consistent change in sensitivity across runs. Figure 4 shows a typical plot of a 13-min criterion run. As can be seen, the threshold value was relatively constant throughout the run. In preliminary studies, both supra- and subthreshold values were used to initiate the run, and the results were virtually the same.

\section{DISCUSSION}

On the basis of these data, it is apparent that temperature and humidity were significant variables in the detection of the flow of nonodorous air in the nasal passages. Basically, the closer the stimuli characteristics were to those of air as conditioned by the nose to nearly saturation and body temperature (Seeley, 1940), the higher the flow threshold. While temperature was an important variable, the uniformly low threshold at RH $5 \%$ implied that the perceived sensation was that of mucosal cooling due to evaporation. Even when the temperature was higher than body temperature, evaporative action

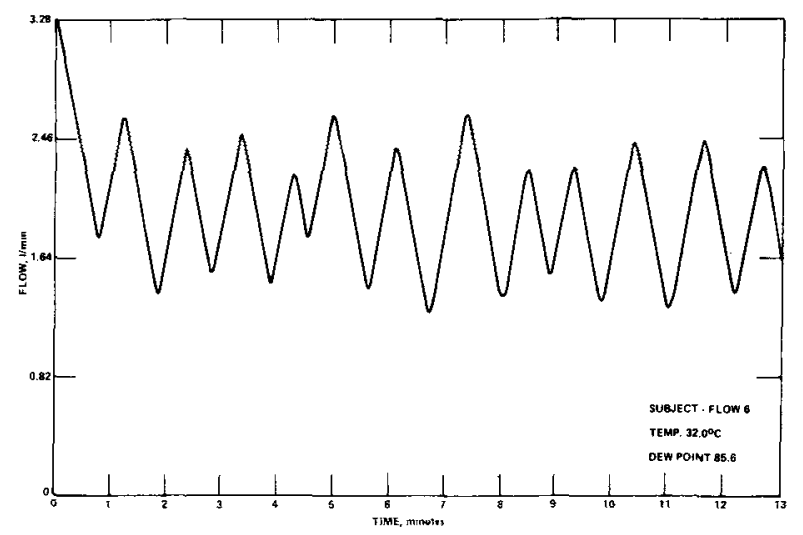

Figure 4. Plot of a representative criterion run. could produce mucosal cooling. This conjecture was reinforced by the introspective reports of the subjects who largely reported a sensation of cooling. Apparently, threshold levels of airflow were not transduced by mechanoreception, since the thresholds were so strongly functions of $\mathrm{RH}$ and temperature.

Although the thresholds observed in this study for nasal airflow are quite low compared with normal resting respiratory flow rates, which are as high as 30 liters/min (Proctor \& Hardy, 1949), Mullins (1955), who employed a flow dilution olfactometer, reported that continuous flows higher than 6 liters/ min were unpleasant and distracting in olfactory experimentation. It may be that a continuously flowing airstream through the nasal passages disrupts the nasal cycle (Stoksted, 1952), giving rise to unpleasant sensations.

It is important to note that the sensory receptors stimulated at threshold levels of flow might be different from those stimulated at higher flow values during sniffing or breathing. As the flow rate increases, it is reasonable to expect that mechanoreceptors may become activated so that the flow sensation experienced will not only be more intense, but will probably also change in character, altering the olfactory Gestalt. Utilizing a subthreshold level of airflow may reduce the contribution of the trigeminal nerve to olfaction. The chemosensory free nerve endings would still be responsive, but the contributions of the thermo- and mechanosensing units would be minimized. Evaluation of the chemical sense might be better at subthreshold flow than at a suprathreshold level.

In this study, special attention must be addressed to the possibility that the airstream was not purified well enough to prevent some contaminant from acting as an olfactory stimulus and thus confounding the result, even though the examination of the filter effluent demonstrated purity. It is almost impossible to ensure that an entirely uncontaminated airstream was available because of the lack of broad-range sensitivity in analytical instruments. Quite possibly, the human sensory system is much more sensitive than analytic instrumentation. Despite efforts to eliminate them, particles of nonodorous materials (Turk \& Bownes, 1951) or aerosols may have provided trigeminal or olfactory stimulus. If particulates provided a detectable sensation, one would not expect such strong temperature and humidity dependence. If aqueous aerosols had provided stimulation, then one would expect a decrease in threshold with an increase in RH. Additionally, uncontrolled olfactory cues would not be expected to exhibit the dramatic humidity dependence seen here, and there is no strong evidence for such an effect (Stone, 1968). For these reasons, the possibility of contamination of the airstream seems unlikely.

There is practical import to these data. In addition 
to the theoretical contribution of the sensation of flow to olfactory perception, it was demonstrated that it is important to control and specify the flow, temperature, and humidity of olfactory stimuli when flow dilution techniques and nasal catheters are employed. This technique, although less commonly used than sniff bottles, has the advantage of stimulus specification. Failure to control these variables could provide nonolfactory cues.

\section{REFERENCES}

Air quality criteria for particulate matter (National Air Pollution Control Administration, Publication No. AP-49). Washington, D.C: U.S. Government Printing Office, 1970.

Bocca, E., \& Battiston, M. N. Odour perception and environmental conditions. Acta Oto-Laryngologica, 1964, 57, 391-400.

DOUEk, E. The sense of smell and its abnormalities. Edinburgh: Churchill Livingstone, 1974.

Grundvig, J. L., Dustman, R. E., \& Beck, E. C. The relationship of olfactory receptor stimulation to stimulus-environmental temperature. Experimental Neurology, 1967, 18, 416-428.

KERKA, W. F., \& HuMPHREYS, C. M. Temperature and humidity effect on odor perception. Heating, Piping, and Air Conditioning, 1956, 28, 129-136.

LeMagnen, J. Étude des facteurs dynamiques de l'excitation olfactive. L'Année Psychologique, 1944-1945, 45-46, 77-89.

Moulton, D. G., \& Tucker, D. Electrophysiology of the olfac- tory system. Annals of the New York Academy of Sciences, $1964,68,380-427$.

Mullins, L. J. Olfaction. Annals of the New York Academy of Sciences, 1955, 62, 247-276.

Proctor, D. F., \& HARdy, J. B. Studies of respiratory air flow. Bulletin of Johns Hopkins Hospital, 1949, 85, 253-280.

REHN, T. Perceived odor intensity as a function of air flow through the nose. Sensory Processes, 1978, 2, 198-205.

Schneider, R. A., Costiloe, J. P., Vega, A., \& Wolf, S. Olfactory threshold technique with nitrogen dilution of n-butane and gas chromatography. Journal of Applied Physiology, 1963, 18, 414-417.

SeELEY, L. E. Study of changes in the temperature and water vapor content of respired air in the nasal cavity. Heating, Piping, and Air Conditioning, 1940, 12, 377-383.

STOKSTED, $P$. The physiologic cycle of the nose under normal and pathologic conditions. Acta Oto-Laryngologica, 1952, 42, 175-179.

STONE, H. Influence of temperature on olfactory sensitivity. Journal of Applied Physiology, 1963, 18, 746-751.

STONE, H. Physiological and psychological aspects of olfactory response in man. In N. N. Tanyolac (Ed.), Theories of odor and odor measurement. Berkshire, England: Unwin Brothers, 1968.

TUCKE R, D. Physical variables in the olfactory stimulation process. Journal of General Physiology, 1963, 46, 453-489.

Turk, A., \& Bownes, K. Inadequate stimulation of olfaction. Science, 1951, 114, 234-236.

(Received for publication April 5, 1979; revision accepted February 22, 1980.) 